\title{
Performance Analysis of Multiresolution Joint Source-Channel Coding for Mobile Radio Channels with feedback
}

\author{
Xufang Wang, Vincent K. N. Lau and T. S. Ng \\ Department of Electrical and Electronic Engineering \\ The University of Hong Kong, Pokfulam Road, Hong Kong \\ Phone: (852) 2857-8408, Fax: (852) 2559-8738 \\ e-mail: xfwang@eee.hku.hk, knlau@eee.hku.hk and tsng@eee.hku.hk
}

\begin{abstract}
In this paper, we analyze the performance of a novel multiresolution joint source and channel coding (MR-JSCC) scheme for mobile radio channels with feedback. The transmitter and receiver can match the resolution of each other synchronously, according to the channel state information (CSI). Bandwidth requirement could be met by carefully adjusting the resolution thresholds. The threshold determination algorithm is outlined. Simulation results show that MR-JSCC scheme has much better performances than baseline-I (No UEP and No feedback) and baseline-II (UEP without feedback) at the same bandwidth and power.
\end{abstract}

\section{INTRODUCTION}

Today, transmission over mobile radio channel is still a great challenge due to the severe impairment caused by shadowing, Rayleigh fading, interference from other uses. In many cases, error correction codes are used to protect the source data from channel errors. Traditionally, fixed-rate error correction coding is used which failed to exploit the time varying nature of the mobile radio channel. Recently, there are a number of adaptive channel coding schemes proposed which could adjust the level of redundancy according to the channel condition with the help of a feedback channel. Significant gains in terms of throughput and SNR are reported in the literatures $[1,2]$.

On the other hand, Shannon's separation principle [3] states that source coding and channel coding can be theoretically separated with no loss in performance for point-to-point communications assuming the use of that arbitrarily long block length source and channel codes and the availability of arbitrarily complicated resources. These two important conditions, however, are apparently not met in practical communication systems. In fact, many examples of joint source and channel coding (JSCC) have shown great performance gains or reduction in complexity [4][5]. In particular, Cover [6] proved that optimal broadcast scenarios are multiresolution and many applications have emerged afterwards. The application of multiresolution joint source-channel coding (MRJSCC) to digital terrestrial broadcasting of high definition television (HDTV) [7] was shown to be efficient enough to replace the traditional single-resolution techniques and a stepwise graceful degradation was obtained. The uses of multiresolution framework for layered video delivery over multicarrier systems revealed substantial gains in deliverable bit rates over optimized TDM-based design [8]. The reconstructed image quality of multiresolution image transmission over time-varying channels attained on the order of $2 \mathrm{~dB}$ gains over single-resolution systems [9]. However, most of these MRJSCC schemes considered only the additive white Gaussian noise (AWGN) channel, which are time invariant. For mobile radio channel, the time varying nature warrant a different system design. Although MRJSCC has been extended to the Rayleigh fading channel recently in [9], no feedback channel has been included in the model and therefore, the system does not effectively exploit the time"varying nature of the mobile radio channels.

In this paper, we propose a novel MRJSCC scheme for mobile radio channels with feedback (both shadowing and Rayleigh fading are considered). We have a multiresolution analog source for transmission. For the channel coding scheme, we adopt a novel channel-adaptive error correction system, namely the Adaptive Bit-Interleaved Coded Modulation (ABICM) [10] which could support an arbitrary number of Unequal Error Protection (UEP) classes with independently controllable BERs for each class. The multiresolution source is jointly and adaptively coupled with the ABICM-UEP scheme according to the current channel state information (CSI). The instantaneous resolution is determined by the CSI together with the resolution thresholds. We investigate the system design issues, the algorithm to determine the resolution threshold as well as the system performance. For the sake of comparison, we considered two baseline systems, namely the baseline-I (No UEP and no feedback) and the baseline-II (UEP and no feedback).

This paper is organized as follows. In section 2, we describe the system model, Based on this model, the MR-JSCC scheme is presented in section 3; simulation results and discussion are given in section 4. Finally, section 5 concludes the paper.

\section{SYSTEM MODEL}

The basic system model employed is illustrated in Figure 
For analytical simplicity, we assumed a Guassian source model although extension to other types of more complicated source model can be made easily. The source coder is a Vector Quantizer (VQ) optimized by the generalized Lloyd algorithm (GLA)[11]. Multiresolution (MR) here refers to multiple codebook resolutions. The VQ indexes, $\left[i_{1}, i_{2}, i_{3}, \ldots i_{N k}\right]$, are protected by the variable rate adaptive bit-interleaved coded modulation (ABICM) [10], which consists of a variable rate convolutional encoder, a bit-by-bit interleaver, a variable throughput adaptive modulator and a channel state predictor. Information bits are convolutionally encoded and the coded bits are bit-interleaved and mapped onto the appropriate modulation symbol. Appropriate encoding modes and modulation constellations are determined symbol-by-symbol at the transmitter based on the feedback channel states. The ABICM scheme is matched with the MR source to provide UEP with adjustable number of classes, the BER level for each class and the throughputs. For a detailed description of the ABICM scheme, please refer to [10].

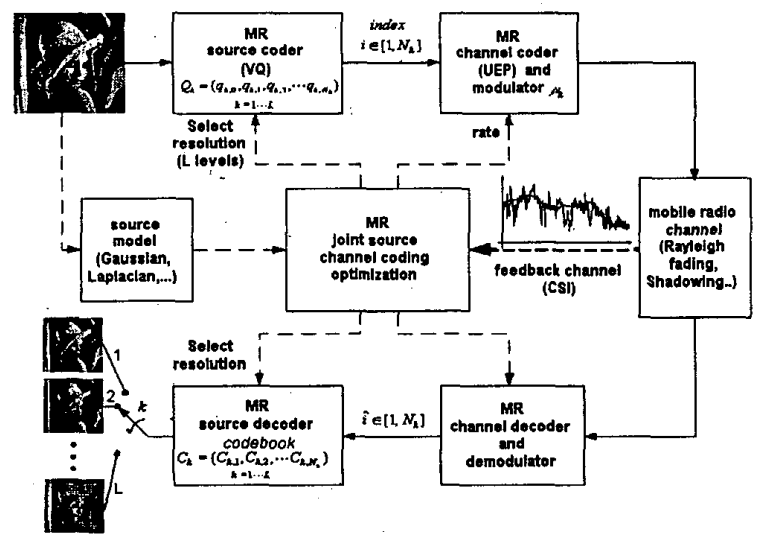

Figure 1: System model for multiresolution joint source and channel coding (MRJSCC) with feedback.

The mobile radio channel is modeled as a superposition of a small-scale quasi-stationary random process (Rayleigh fading) and a large-scale random process (shadowing) [12]. The fading envelope can be represented as follows:

$$
\alpha(t)=s(t) r(t)
$$

where $s(t)$ represents the shadowing component and $r(t)$ represents the short-term fading component. The short-term fading component, $r(t)$, is a Rayleigh distributed stationary random process with coherence time given by:

$$
T_{c}=\frac{\lambda}{v_{d}}
$$

where $\lambda$ is the wavelength and $v_{d}$ is the mobile velocity. For pedestrian to vehicular speed, the coherence time is of the order of milli-seconds. Hence, fast fading component is handled by the ABICM adaptive coding mechanism. The shadowing component, $s(t)$, is a long-term random process which is modeled by the log-normal distribution. The coherence time is of the order of seconds. Therefore, the long-term shadowing is handled by the multi-resolution source and channel encoder matching.

The ABICM is operated in constant BER control where the achieved average -throughput is varied according to the shadowing component $s(t)$ to maintain the target BER level for different UEP classes. The ABICM could provide nearly constant error protection as required over a wide range of shortterm average channel signal to noise (SNR) under Rayleigh fading as illustrated in figure 2 . The resolution mode of the source is selected according to the current short-term average channel SNR, which raisés the interesting question of how to optimally determine the thresholds for different resolution modes given certain bandwidth constraint. The core function box in the block diagram is the MR-JSCC optimization taking both advantages of MR and JSCC.

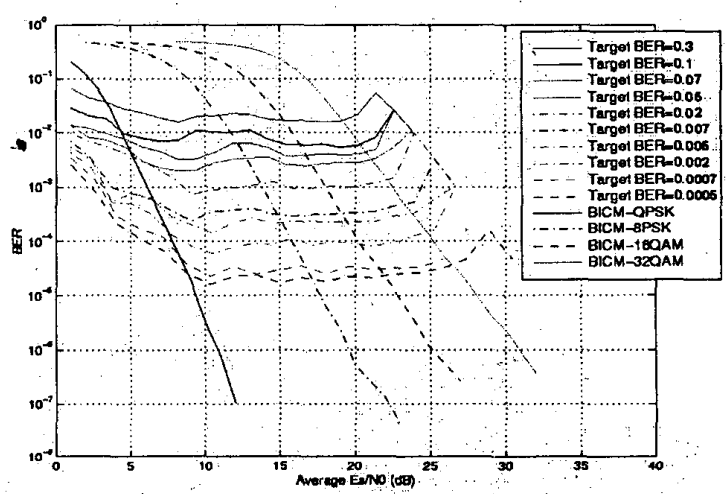

Figure 2: BER against SNR curves of ABICM [10]

\section{PROPOSED FRAMEWORK}

The end-to-end distortion of the MR-JSCC system is related to source MR levels (quantization noise) and ABICM MR modes, which could be expressed as follows:

$$
\begin{aligned}
D_{\text {overall }} & =\int_{s} p(s) E\left[\left.(x-\hat{x})^{2}\right|_{s}\right] d s \\
' & =\sum_{l=0}^{L-1} \int_{S_{i}} p(s) \sum_{i, j=1}^{N_{1}} p_{j l i}(s)\left[\int_{h_{i-1}^{l}}^{l_{i}^{\prime}} P(x)\left(x-c_{l, j}\right)^{2} d x\right] d s
\end{aligned}
$$

where

$P(x)$ is the probability density function (p.d.f.) of the source;

$p_{j \mid i}(s)$ is the probability of receiving $\mathrm{j}$ when $\mathrm{i}$ is transmitted;

$p(s)$ is the p.d.f. of the mobile radio channel; 
$l$ is the label for the resolution levels;

$q_{i}$ is the quantization threshold for the $\mathrm{l}_{\text {th }}$ level resolution $(l=0 . . L-I)$;

$S_{l}$ is the regime of channel SNR that $\mathrm{l}_{\text {th }}$ level resolution is chosen, how to determine $S_{l}$ will be given later in this section.

Equation (1) could be further simplified as:

$D_{\text {overall }}=D_{Q_{l}}+D_{C h_{l}}$

where

$D_{Q_{1}}=\sum_{i=0}^{L-1} \int_{S_{1}} p(s)\left[\sum_{i=1}^{N_{i}} \int_{q_{i-1}^{l}}^{q_{i}^{l}} p(x)\left(x-c_{l, i}\right)^{2} d x\right] d s$

$D_{C_{i}}=\sum_{i=0}^{L-1} \int_{S_{1}} p(s) \sum_{i, j=1 \& i \neq j}^{N_{l}} p_{j l i}(s)\left[\int_{q_{i-1}^{i}}^{q_{i}^{i}} p(x) d x\right]\left(c_{l, i}-c_{l, j}\right)^{2} d s$

Here, $D_{Q !}$ represents the quantization noise, $D_{C h l}$ represents the contribution solely due to channel errors. The mutual error term could be shown to be zero as long as the quantization are optimally performed. Our proof is an extension based on [13].

According to our system model, the total end to end distortion could be represented by

$$
\begin{aligned}
\varepsilon^{2}= & E d\left(\mathbf{X}-\mathbf{C}_{*}\right) \\
= & E\left[\left(\mathbf{X}-\mathbf{C}_{*}\right) \cdot\left(\mathbf{X}-\mathbf{C}_{*}\right)^{t}\right] \\
= & E\left[(\mathbf{X}-\mathbf{C}) \cdot\left(\mathbf{X}-\mathbf{C}^{t}\right]+E\left[\left(\mathbf{C}-\mathbf{C}_{*}\right) \cdot\left(\mathbf{C}-\mathbf{C}_{*}\right)^{t}\right]\right. \\
& +2 E\left[(\mathbf{X}-\mathbf{C}) \cdot\left(\mathbf{C}-\mathbf{C}_{*}\right)^{t}\right] \\
\cdot= & \varepsilon_{q}^{2}+\varepsilon_{*}^{2}+2 E\left[(\mathbf{X}-\mathbf{C}) \cdot\left(\mathbf{C}-\mathbf{C}_{*}\right)^{t}\right]
\end{aligned}
$$

Where

$\mathbf{X}$ is the data vector we want to transmit,

C is the encoded codebook vector,

C* is the decoded codebook vector.

The last term at the right side of the equation may be extended to yield

$E\left[(\mathbf{X}-\mathbf{C})\left(\mathbf{C}-\mathbf{C}_{*}\right)^{t}\right]$

$=E\left[\mathbf{X C}^{t}\right]-E\left[\mathbf{X C}_{*}^{t}\right]-E\left[\mathbf{C C}^{t}\right]+E\left[\mathbf{C C}_{*}^{t}\right]$

For a given partition $\left\{\mathrm{R}_{i} ;=1, \ldots, \mathrm{N}\right\}$,

$p_{i}(\mathbf{x})=\int_{\mathbf{x} \in R_{t}} f(\mathbf{x}) d \mathbf{x}$

Where $f(\mathbf{x})$ is the (joint) pdf of the vector $\mathbf{X}$, and the integration above is understood to be a multiple integral over the $k$ dimensional space.

The conditional probability is given by

$P_{i j}=P\left[\mathrm{c}_{*}=\mathrm{c}_{\mathrm{j}} \mid \mathrm{c}=\mathrm{c}_{\mathrm{j}}\right]$

Then the various joint probability density functions could be calculated as follows:

$$
\begin{aligned}
& f_{r}(\mathbf{x}, \mathbf{c})=\sum_{i=1}^{N} \delta\left(\mathbf{c}-\mathbf{c}_{i}\right) p_{i}(\mathbf{x}) \\
& f_{2}\left(\mathbf{x}, \mathbf{c}_{*}\right)=\sum_{j=1}^{N} \delta\left(\mathbf{c}_{*}-\mathbf{c}_{i}\right) \sum_{j=1}^{N} p_{j}(\mathbf{x}) P_{j i} \\
& f_{3}\left(\mathbf{c}, \mathbf{c}_{*}\right)=\sum_{i=1}^{N} \sum_{j=1}^{N} p_{i}(\mathbf{x}) P_{j i} \delta\left(\mathbf{c}-\mathbf{c}_{i}\right) \delta\left(\mathbf{c}_{*}-\mathbf{c}_{j}\right)
\end{aligned}
$$

$$
f_{4}(\mathbf{c})=\sum_{i=1}^{N} p_{i}(\mathbf{x}) \delta\left(\mathbf{c}-\mathbf{c}_{1}\right)
$$

Therefore

$$
\begin{aligned}
& E\left[\mathbf{X C}^{t}\right]=\sum_{i=1}^{N}\left[\int_{\mathbf{x} \in R_{i}} \mathbf{x} f(\mathbf{x}) d \mathbf{x}\right] \cdot \mathbf{c}_{i}^{t} \\
& E\left[\mathbf{C C}^{t}\right]=\sum_{i=1}^{N} \mathbf{c}_{i} \mathbf{c}_{i}^{t} p_{i}(\mathbf{x}) \\
& E\left[\mathbf{C C}^{t}\right]=\sum_{i=1}^{N} \sum_{j=1}^{N} \mathbf{c}_{i} \mathbf{c}_{j}^{t} p_{i}(\mathbf{x}) P_{i j} \\
& E\left[\mathbf{X C}_{i}^{t}\right]=\sum_{i=1}^{N}\left[\sum_{j=1}^{N} P_{j i} \int_{\mathbf{x} \in R_{i}} \mathbf{x f}(\mathbf{x}) d \mathbf{x}\right] \cdot \mathbf{c}_{i}^{t}
\end{aligned}
$$

When the vector quantization is optimally performed, the code vectors must be the centroid [11]

$\mathbf{c}_{i}=\operatorname{cent}\left(R_{i}\right)=\frac{\int_{\mathbf{x} \in R_{i}} \mathbf{x} f(\mathbf{x}) d \mathbf{x}}{\int_{\mathbf{x} \in R_{i}} f(\mathbf{x}) d \mathbf{x}}$

Substituting (4), (5) and (14) to (10)-(13), we get

$E\left[(\mathbf{X}-\mathbf{C})\left(\mathbf{C}-\mathbf{C}_{*}\right)^{t}\right]=0$

Hence

$$
\varepsilon^{2}=\varepsilon_{q}^{2}+\varepsilon_{*}^{2}
$$

whenever the quantization are optimally performed.

As the wireless channel is always fluctuating due to fading, the allocation of the bits in the source and the channel coding, therefore, should adapt to it. When the channel condition is good, more bits should be allocated to the source, which can now afford high level of resolution, and fewer bits are spent for error protection. Otherwise, more bits should be used for error protection to guarantee acceptable BER, so the resolution level of the source has to be reduced. A fairly good criterion [4] of bit allocation between source and channel coding is such that

$$
D_{Q_{i}}=D_{C_{h_{l}}}
$$

We assume the error protection is strong enough that single bit error is the most likely to happen. Let $\overline{P_{b n i}}(s)$ denote the average BER for the $\mathrm{m}_{\mathrm{th}}$ bit, then $D_{C h l}$ can be further simplified as below:

$$
D_{C c_{q}} \approx \sum_{l=1}^{L-1} \int p(s) \sum_{i=1}^{N_{1}} \sum_{m=1}^{M_{l}} \overline{p_{b m !}}(s) \overline{\beta_{m !}} d s
$$

Where

$\overline{\beta_{m, l}}=\sum_{i=1}^{N_{1}}\left(c_{l, i}^{\prime}-c_{l, i e m}\right)^{2} \int_{q,-1}^{q_{i}^{l}} p(x) d x$

is the average bit error sensitivity for the $m_{\mathrm{th}}$ bit.

We adopt the intuitive rule (also adopted in [4]) that the contribution of all the bits after error protection to the average overall noise caused by the transmission errors should be equal. Moreover, the error protection provided by the channel code is required to match to the error sensitivity of the source bits for the efficient use of the limited channel bandwidth. Best 
protection is given to those bits which are the most sensitive to channel errors. Thus an effective unequal error protection is achieved by letting the error protection profile be such that

$\overline{p_{b m, l}}(s) \overline{\beta_{m, l}}=$ const,$\quad m=1 \cdots M_{l}$

After combining equation (15) and (18), the bit error protection for every bit of VQ indexes of each MR level could be obtained.

When the channel state is bad, we pick low resolution mode which means fewer information bits from the source and more redundancy bits for protection. Therefore, the overall throughput is low. As the channel condition gets better, higher resolution mode is selected, resulting in more information bits from the source and less redundancy bits for protection. Hence, a higher throughput is achieved. In the proposed MR-JSCC system, we have $M_{1}$ resolution levels. Resolution $m$ is chosen if the short-term average SNR, $s(t) \in\left[\xi_{m-1}, \xi_{m}\right]$ where $\xi_{m}$ is the resolution thresholds.

\section{Determination of Resolution Thresholds}

The resolution thresholds are critical to the performance of the MR-JSCC system. In this section, we propose an algorithm to obtain the thresholds. The thresholds should be set with a constant bandwidth constraint given by:

$$
\sum_{i=0}^{4} \frac{n_{i+1}}{\int_{s_{1}}^{h_{1+1}} \rho(i+1, s) f(s) d s}=N_{c}=\text { const. }
$$

Where, $n_{i+1}$ is the number of information bits [resolution mode $i+1]$ per frame, $n_{i}<n_{i+1} ; N_{c}$ is the number of symbols per frame; $\rho(i+1, s)$ is the average throughput of resolution mode $i+1$ under the average SNR $s ; f(s)$ is the p.d.f. of the log-normal shadowing; $\xi_{i}$ is the threshold, $i=0$ to $5, \xi_{0}=0, \xi_{5}=\infty$.

The algorithm of the threshold determination is outlined as follows.

Step 1. given the appropriate value of $\mathrm{N}_{c}$;

Step 2. find out $\xi_{1}$ by equation(19);

Step 3. calculate the rest of thresholds $\xi_{2}, \xi_{3}, \xi_{4}$ in the same way. If some of the thresholds are out of range, the value of $\mathrm{N}_{\mathrm{c}}$ could be adjusted to accommodate more resolution modes.

\section{SIMULATION RESULTS AND DISCUSSION}

Fig 3 shows the end-to-end distortion after applying the MR-JSCC scheme to the Guassian source and mobile radio channel. The average throughput of MR-JSCC is increasing with the increase of the long-term average SNR as expected (Fig 4). We have achieved nearly constant bandwidth by carefully adjusting the thresholds according to the threshold determination algorithm, which is illustrated in Fig 5.

To illustrate the significance of MR-JSCC system with feedback, we consider. two reference systems at the same bandwidth for comparison. They are namely the Baseline-I and
Baseline-II systems. Baseline-I consists of a single resolution source, a channel encoder without UEP and mobile radio channel without feedback. Baseline-II consists of a single resolution source, a channel encoder with UEP but without feedback channel. As we can see clearly from the figure, MRJSCC has achieved substantial gains in distortion comparing to the two reference systems. This illustrates the benefit of employing a multi-resolution approach with feedback channel. At low SNR, the overall distortion is dominated by the channel error for the two baseline systems. At high SNR, the overall distortion is dominated by the quantization noise for the two baseline systems. For the proposed MR-JSCC system with feedback, the system is able to adjust the optimal resolution according to the variation of SNR. Therefore, a balance between channel errors and quantization noise is always achieved, resulting in a better distortion performance compared with the baseline systems.

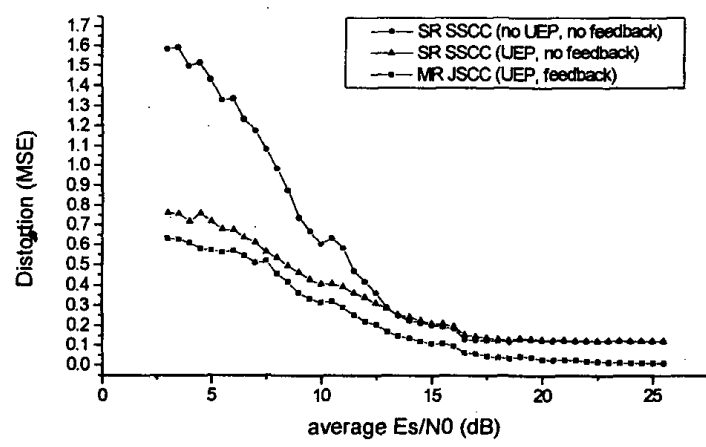

Figure 3: The end-to-end distortion versus average channel SNR curves for MR-JSCC and two reference respectively.

\section{CONCLUSION}

We have proposed a multiresolution joint source and channel coding (MR-JSCC) for mobile radio channels with feedback. The transmitter and the receiver selected the resolution mode synchronously according to the average channel SNR and the thresholds for different resolution modes could be adjusted to meet the bandwidth requirement. Simulation results show that MR-JSCC performs better than two reference systems (no feedback with or without UEP). Future work would be applying this scheme to image transmission, which is currently under way.

\section{REFERENCES}

[1] S. Alamouti and S. Kallel, "Adaptive Trellis-Coded Multiple-Phase-Shift Keying for Rayleigh Fading Channels," IEEE Trans. On Communs., vol. 42, pp. 23052314, June 1994.

[2] K. N. Lau and S. Maric, "Adaptive Non-coherent M-ary Orthogonal Modulation with RAKE Receiver for DSCDMA," IEEE Trans. On Communications., April 1999. 
[3] C. E. Shanron, "A mathematical theory of communication," Bell Syst. Tech. Journal, vol.27, pp. 379-423, 1948.

[4] R. V. Cox, J. Hagenauer, and C. W. Sundberg, "Subband speech coding and matched convolutional Channel Coding for mobile radio channels," IEEE Trans. Signal Processing, vol. 39, No. 8, pp. 1717-1731, August 1991.

[5] A. J. Goldsmith and M. Effros, "Joint design of fixed-rate source codes and multiresolution channel codes", IEEE Trans. communications, vol.46, no10, pp. 1301-1312, Oct 1998

[6] T. Cover, "Broadcast channels," IEEE Trans. Inform. Theory, vol. IT-18, pp. 2-14, Jan. 1972.

[7] K.Ramchandran, A. Ortega, K. M. Uz, and M. Vetterli, "Multiresolution broadcast for digital HDTV using joint source-channel coding," IEEE J. Select. Areas Commun., vol. 11, pp. 6-23, Jan. 1993.

[8] S. radhan and K.Ramchandran, "Efficient layered video delivery over multicarrier systems using optimized embedded modulation," in Proc. IEEE int. Conf. Imag. Process., vol.3, pp.452-455, 1997,

[9] I. Kozintsev and K. Ramchandran, "Robust image transmission over energy-constraint time varying channels using multiresolution joint source-channel coding, " IEEE Trans. Signal Processing, vol. 46, pp.1012-1026, April 1998.

[10] V. K. N. Lau, "On the design of variable rate adaptive bit interleaved coded modulation," Proceedings of IEEE VTC2000, May 2000, Tokyo.

[11] A. Gersho and R. M. Gray, Vector quantization and Signal Compression. Norway, MA: Kluwer, 1992.

[12] Cox, D.C., Murray, R. R., and Norris, A. W., " $800 \mathrm{MHz}$ attenuation measured in and around suburban houses," AT\&T Bell Lab. Tech. Journal, vol.673, No.6, pp. 921954, July-August, 1984.

[13] R. E. Totty and G.C. Clark, "Reconstruction error in waveform transmisstion," IEEE Trans. Inform. Theory, vol. IT-13, pp.336-338, 1967.

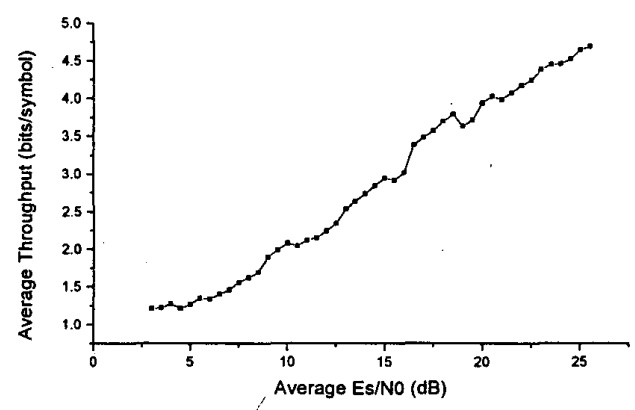

Figure 4: Average throughput versus average channel Es/ $\mathrm{N}_{0}$ curve of MR-JSCC

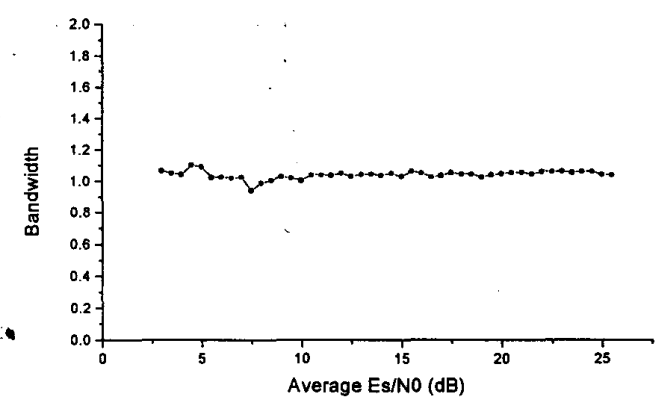

Figure 5: Bandwidth versus average channel $\mathrm{Es} / \mathrm{N}_{0}$ curve of MR-JSCC 